\title{
The Role of Scalar Mesons in Extended Perturbation Scheme for Effective Field Theory
}

\author{
Kirill Semenov-Tian-Shansky*, Alexander Vereshagin ${ }^{\dagger}$ and Vladimir \\ Vereshagin** \\ *Institut für Theoretische Physik II, Ruhr-Universität Bochum, Bochum, Germany \\ ${ }^{\dagger}$ Department of Physics and Technology, University of Bergen, Bergen, Norway \\ ${ }^{* *}$ Department of High Energy Physics, Saint-Petersburg State University, Saint-Petersburg, Russia
}

\begin{abstract}
We argue how it is possible to apply the general scheme of the effective scattering theory (EST) to the description of the hadronic processes. The results of the numerical tests of sum rules for $\pi N$ spectrum parameters that follow from the bootstrap system allow us to claim the consistency of the predictions obtained in the framework of our approach with the known phenomenology. We also demonstrate that the tree-level low energy expansion coefficients computed in the framework of our approach show nice agreement with known experimental data.
\end{abstract}

Keywords: Effective field theories, renormalizability

PACS: $02.30 . \mathrm{Lt}, 11.15 . \mathrm{Bt}, 13.75 . \mathrm{Gx}, 14.20 . \mathrm{Gk}$

\section{INTRODUCTION}

In papers $[3,4,5,6]$ we started the systematic study of the special class of effective theories of strong interaction. Our principle goal is to develop a self consistent Dyson perturbation technique for the infinite component effective scattering theory of strong interaction. It is quite reasonable to start from the definition of such a theory. We use a slightly modified version of the definition first given in [1]. The field theory is called effective if the quantum interaction Hamiltonian (in the interaction picture) contains all the monomials consistent with a given algebraic (linear) symmetry. The effective theories are as renormalizable as the ordinary renormalizable ones. The only difference is that one needs to formulate an infinite number of renormalization prescriptions (RPs) fixing the finite part of counterterms. Effective theories are intrinsically quantum constructions since we relay upon Weinberg scheme of constructing QFT (see e.g. [2]). This approach is adjusted for $S$-matrix calculations. We call a theory constructed with the use of this scheme as effective scattering theory (EST).

In our approach we deal only with a very narrow class of localizable effective scattering theories and introduce the notion of extended perturbative scheme (see the discussion in $[3,5])$. The hypothetical localizable effective theory of strong interaction requires an infinite extension of perturbative scheme by introduction of an infinite tower of baryon and meson resonances of arbitrary high spin and mass. When dealing with such a theory one has first to point out a way to assign meaning to the perturbation series. The second problem is to somehow reduce the number of independent parameters for which it is necessary to formulate RPs fixing the physical contents of the theory. In Refs. [3, 4, 5] we propose a way to construct the meaningful perturbative scheme for such a theory. 


\section{CONSTRUCTION OF THE CAUCHY FORMS}

The amplitude $M_{a \alpha}^{b \beta}$ of $\pi N$ binary scattering

$$
\pi_{a}(k)+N_{\alpha}(p, \lambda) \rightarrow \pi_{b}\left(k^{\prime}\right)+N_{\beta}\left(p^{\prime}, \lambda^{\prime}\right)
$$

can be presented in the following form (isotopic invariance is taken to be the exact symmetry of strong interaction):

$$
M_{a \alpha}^{b \beta}=\delta_{b a} \delta_{\beta \alpha} M^{+}+i \varepsilon_{b a c}\left(\sigma_{c}\right)_{\beta \alpha} M^{-}
$$

where

$$
M^{ \pm}=\bar{u}\left(p^{\prime}, \lambda^{\prime}\right)\left\{A^{ \pm}+\left(\frac{\hat{k}+\hat{k}^{\prime}}{2}\right) B^{ \pm}\right\} u(p, \lambda)\left(\hat{k} \equiv \gamma_{\mu} k^{\mu}\right) .
$$

The invariant amplitudes $A^{ \pm}, B^{ \pm}$are certain functions of Mandelstam variables $s, t, u$.

The tree-level binary $\pi N$ scattering amplitude calculated in the framework of our effective theory approach is the sum of all possible $s-, t$ - and $u$-channel resonance exchanges plus the sum of contributions of all possible $\pi \pi N \bar{N}$ vertices. To assign meaning to this sum (which is certainly a formal one) one has to switch to minimal parametrization (see [4, 5]) and to use the method of Cauchy forms ([3] and Refs. therein). The transition to the minimal parametrization helps to get rid of those combinations of Hamiltonian couplings which appear only in off-shell matrix elements and hence does not require the formulation of RPs since we are only interested in the calculation of the $S$-matrix. In the minimal parametrization the vertices (Hamiltonian monomials) we need are the following:

$$
\begin{aligned}
& J=l+\frac{1}{2}(l=0,1,2 \ldots) \\
& I=\frac{1}{2}, \mathscr{N}=-1: \quad g_{\widehat{R} \pi N} \bar{N} \sigma \widehat{R}_{\mu_{1} \ldots \mu_{l}} \partial^{\mu_{1}} \ldots \partial^{\mu_{l}} \pi+H . c . \\
& I=\frac{1}{2}, \mathscr{N}=+1: \quad i g_{R \pi N} \bar{N} \sigma \gamma_{5} R_{\mu_{1} \ldots \mu_{l}} \partial^{\mu_{1}} \ldots \partial^{\mu_{l}} \pi+H . c . \\
& I=\frac{3}{2}, \mathscr{N}=-1: \quad g_{\widehat{\Delta} \pi N} \bar{N} P_{\frac{3}{2}} \widehat{\Delta}_{\mu_{1} \ldots \mu_{l}} \partial^{\mu_{1}} \ldots \partial^{\mu_{l}} \pi+H . c .
\end{aligned}
$$

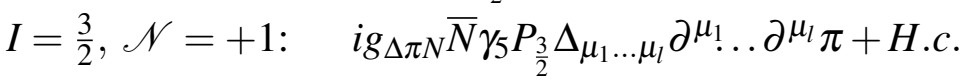

$$
\begin{aligned}
& J=0,2, \ldots, I=0, P=+1 \text { : } \\
& \frac{1}{2} g_{S \pi \pi} S_{\mu_{1} \ldots \mu_{J}}\left(\pi \cdot \partial^{\mu_{1}} \ldots \partial^{\mu_{J}} \pi\right) \\
& {\left[g_{N N S}^{(1)} \bar{N} \partial_{\mu_{1}} \ldots \partial_{\mu_{J}} N+i g_{N N S}^{(2)} J \partial_{\mu_{1}} \ldots \partial_{\mu_{J-1}} \bar{N} \gamma_{\mu_{J}} N\right] S^{\mu_{1} \ldots \mu_{J}} \text {, }} \\
& J=1,3, \ldots, I=1, P=-1 \text { : } \\
& \frac{1}{2} g_{V \pi \pi} V_{\mu_{1} \ldots \mu_{J}}\left(\pi \times \partial^{\mu_{1}} \ldots \partial^{\mu_{J}} \pi\right) \\
& {\left[i g_{N N V}^{(1)} \bar{N} \sigma \partial_{\mu_{1}} \ldots \partial_{\mu_{J}} N+g_{N N V}^{(2)} J \bar{N} \gamma_{\mu_{J}} \sigma \partial_{\mu_{1}} \ldots \partial_{\mu_{J-1}} N\right] V^{\mu_{1} \ldots \mu_{J}} \text {. }}
\end{aligned}
$$

Here $g_{\ldots}^{\cdots} \equiv g_{\ldots}^{\cdots}(J, I, P)$ stand for real coupling constants, and $\sigma_{c}(c=1,2,3)$ are Pauli matrices. $\mathscr{N}$ stands for resonance normality $\left(\mathscr{N} \equiv P(-1)^{l}\right)$, where $P$ is parity. $P_{\frac{3}{2}} \equiv$ $\left(P_{\frac{3}{2}}\right)_{a \alpha b \beta}$ is the projecting operator on the states with isospin $I=3 / 2, a, \alpha, b, \beta$ being the isotopic indices. 
To construct the Cauchy forms for tree-level binary scattering amplitudes one needs to fix the values of the residues at the relevant poles and to choose properly the bounding polynomial degree. Residues at poles of tree-level amplitudes are just the on-shell resonance spin sums for dotted by the minimal triple coupling constants. It is at this step that we take the main advantage of minimal parametrization since there is only a finite number of minimal triple vertices for each resonance with given quantum numbers. The bounding polynomial degrees are chosen in accordance with the known values of corresponding Regge intercepts.

This results in uniformly converging series of singular terms defining tree-level amplitude as the polynomially bounded meromorphic function in three mutually intersecting layers $B_{x}:\left(x \in R, x \sim 0 ; \quad v_{x} \in C\right)$, where $x=s, t, u$ and $v_{x}, x(x=s, t, u)$ : $v_{s}=u-t, \quad v_{t}=s-u, \quad v_{u}=t-s$. fixing the invariant amplitude in the layer up to few unknown functions.

One of the principal results of [5] states that if one relays upon the renormalized perturbation theory scheme with on-shell renormalization point it is sufficient to formulate RPs only for minimal triple couplings and (real) resonance masses. The next step is to show that although the number of RPs fixing the physical contents of EST is still infinite these RPs are not independent.

\section{BOOTSTRAP SYSTEM}

Bootstrap system arise as the requirement that the Cauchy forms (different in different layers) should coincide in the domains of intersection of layers. This system constrains the allowed values of fundamental observables of the theory (triple minimal couplings and mass parameters). Besides it completely determines the allowed form of the fourleg pointlike vertex contributions and in this way helps to fix completely the binary scattering amplitude.

For example the set of bootstrap constrains for $A^{-}$in $B_{t} \cap B_{u}:\{t, u \in R ; t \sim 0, u \sim 0\}$ domain reads:

$$
\Psi_{s}\left(A^{-}\right) \equiv\left[\text { Cauchy form in } B_{u}\right]-\left[\text { Cauchy form in } B_{t}\right]=0 \text { for } t, u \sim 0 .
$$

Expanding the bootstrap equation in powers of kinematical variables $t, u$ in the vicinity of $(t=0, u=0)$ one obtains an infinite set of sum rules for minimal (resultant) triple couplings and resonance masse parameters. These constrains $(m, n=0,1, \ldots)$ read as:

$$
\sum_{\text {Baryons }} g_{R_{B} \pi N}^{2} V_{m, n}\left(M_{R_{B}}, J, \mathscr{N}, I\right)-\sum_{\substack{\text { Mesons with } \\ \mathrm{I}=1, \text { odd } \mathrm{J}=-\mathrm{P}=-1}} g_{R_{M} \pi \pi} \cdot g_{R_{M} N \bar{N}} W_{m, n}\left(M_{R_{M}}, J\right)=0 .
$$

Here $g_{R_{B} \pi N}\left(g_{R_{M} \pi \pi}, g_{R_{M} N \bar{N}}\right)$ stand for minimal triple couplings of baryon (meson resonances) with pions and nucleons. $V_{m, n}$ and $W_{m, n}$ are certain known functions depending on resonance quantum numbers (mass parameter, spin, normality and isospin). Bootstrap constrains are renorm-invariant in the sense that they are the equations for physical renormalization prescriptions (RPs): triple couplings and mass parameters.

Since bootstrap constrains connect physical quantities the sum rules (6) can be checked directly with the help of experimental data. Large series of well saturated sum 
rules can be derived for the invariant amplitude $A^{-}$as well as for other amplitudes (see [6]). This shows that the system of postulates used in our EST approach looks quite reasonable.

\section{LOW ENERGY COEFFICIENTS}

Here we present our estimates for the expansion coefficients of tree level amplitudes around the cross-symmetric point $\left(t=0, v_{t}=0\right)$ in $B_{t}$ layer. These results present certain interest because those coefficients undoubtedly do acquire contributions from the loop graphs. Nevertheless, as shown below, our estimates based on the tree level approximation of extended perturbation scheme turn out to be in nice agreement with the known data. This fact demonstrates that the latter scheme provides quite reasonable numbers already at tree level and, hence, may be of interest from the computational point of view.

Introducing the new quantity

$$
C^{ \pm}=A^{ \pm}+\frac{m v_{t}}{4 m^{2}-t} \tilde{B}^{ \pm}
$$

(here $\tilde{B}^{ \pm}$is just $B^{ \pm}$with the nucleon pole subtracted) we define the low-energy coefficients (LEC's) $a_{m n}^{ \pm}, b_{m n}^{ \pm}$, and $c_{m n}^{ \pm}$as those in double Taylor series expansions around the cross-symmetric point $\left(t=0, v_{t}=0\right)$ :

$$
\begin{aligned}
& \tilde{B}^{+}\left(t, v_{t}\right)=v_{t} \sum_{m, n} b_{m n}^{+}\left(v_{t}^{2}\right)^{m} t^{n} ; \quad \tilde{B}^{-}\left(t, v_{t}\right)=\sum_{m, n} b_{m n}^{-}\left(v_{t}^{2}\right)^{m} t^{n} ; \\
& C^{+}\left(t, v_{t}\right)=\sum_{m, n} c_{m n}^{+}\left(v_{t}^{2}\right)^{m} t^{n} ; \quad C^{-}\left(t, v_{t}\right)=v_{t} \sum_{m, n} c_{m n}^{-}\left(v_{t}^{2}\right)^{m} t^{n}
\end{aligned}
$$

To get numerical values for these coefficients, we need to re-expand the Cauchy forms in double power series in $\left(t, v_{t}\right)$. This is quite admissible because these forms converge uniformly in whole $B_{t}$ and, therefore, near the cross-symmetric point.

Using the explicit form of bootstrap constrains one can check that mesons with $I=J=0$ make contribution to the smooth (background) part of the amplitude $A^{+}$(and hence $C^{+}$) in the layer $B_{t}$. The Table 1 shows that the experimentally known values of $c_{00}^{+} \div c_{02}^{+}$(see [7]) cannot be explained if we neglect the contribution due to light scalar $\sigma$-meson with the mass parameter $M_{\sigma} \sim 550 \div 700 \mathrm{MeV}$ and "effective coupling" (see [7])

$$
G_{1}^{0} \equiv g_{S \pi \pi} g_{N N S}^{(1)} \sim 50 \div 100
$$

(this statement remains true with respect to $a_{03}^{+}\left(c_{03}^{+}\right)$). Altogether, these results show that the extended perturbation scheme provides reasonable values for the low energy coefficients already at tree level. We emphasize that this is closely connected with the postulated Regge asymptotic conditions in the hyperlayer $B_{t}$. 
TABLE 1. Tree level low energy coefficients $c_{m n}^{+}(m, n=0,1,2) ; X^{N} \equiv X \times 10^{N}$

\begin{tabular}{l|llllllll|l|l|} 
Resonance & $c_{00}^{+}$ & $c_{01}^{+}$ & $c_{02}^{+}$ & $c_{10}^{+}$ & $c_{11}^{+}$ & $c_{12}^{+}$ & $c_{20}^{+}$ & $c_{21}^{+}$ & $c_{22}^{+}$ \\
\hline$\sigma(650)$ & +19 & +0.94 & $+5.20^{-2}$ & & & & & \\
\hline$\Delta(1232)$ & $\begin{array}{l}+2.74 \\
\pm 4.6^{-1}\end{array}$ & $\begin{array}{l}+7.18^{-1} \\
\pm 1.2^{-2}\end{array}$ & $\begin{array}{l}-1.66^{-2} \\
\pm 2.8^{-4}\end{array}$ & $\begin{array}{l}+1.17 \\
\pm 9.8^{-2}\end{array}$ & $\begin{array}{l}+1.68^{-1} \\
\pm 1.4^{-3}\end{array}$ & $\begin{array}{l}-8.56^{-3} \\
\pm 4.6^{-5}\end{array}$ & $\begin{array}{l}+2.12^{-1} \\
\pm 1.8^{-2}\end{array}$ & $\begin{array}{l}+2.38^{-2} \\
\pm 6.4^{-4}\end{array}$ & $\begin{array}{l}-2.37^{-3} \\
\pm 1.5^{-5}\end{array}$ \\
\hline \multirow{2}{*}{$N(1440)$} & -3.86 & $+4.50^{-2}$ & $-3.76^{-4}$ & $+6.65^{-2}$ & $+3.02^{-3}$ & $-6.37^{-5}$ & $+3.35^{-3}$ & $+9.59^{-5}$ & $-5.04^{-6}$ \\
& \pm 1.6 & $\pm 1.9^{-2}$ & $\pm 1.6^{-4}$ & $\pm 1.3^{-1}$ & $\pm 2.0^{-3}$ & $\pm 2.4^{-5}$ & $\pm 6.4^{-3}$ & $\pm 1.7^{-4}$ & $\pm 3.1^{-6}$ \\
\hline \multirow{2}{*}{ Full set } & +23.1 & +1.63 & $+3.50^{-2}$ & +1.39 & $+1.83^{-1}$ & $-8.41^{-3}$ & $+2.19^{-1}$ & $+2.40^{-2}$ & $-2.37^{-3}$ \\
& \pm 6.6 & $\pm 1.2^{-1}$ & $\pm 1.7^{-3}$ & $\pm 4.3^{-1}$ & $\pm 7.9^{-3}$ & $\pm 1.2^{-4}$ & $\pm 2.8^{-2}$ & $\pm 8.6^{-4}$ & $\pm 1.9^{-5}$ \\
\hline \hline \multirow{2}{*}{ Data } & +25.6 & +1.18 & $+3.55^{-2}$ & +1.18 & $+1.53^{-1}$ & $-1.50^{-2}$ & $+2.00^{-1}$ & $+3.40^{-2}$ & $-8.00^{-3}$ \\
& $\pm 5.0^{-1}$ & $\pm 5.0^{-2}$ & $\pm 7.0^{-3}$ & $\pm 5.0^{-2}$ & $\pm 1.7^{-2}$ & $\pm 3.0^{-3}$ & $\pm 1.0^{-2}$ & $\pm 1.0^{-3}$ & $\pm 1.0^{-3}$ \\
\hline
\end{tabular}

\section{CONCLUSIONS}

We develop the logically complete scheme of EST suitable for the description of hadronic scattering processes. Numerical test of sum rules for $\pi N$ (and also $\pi \pi$ and $K N$ ) resonance parameters show that the system of postulates forming the basis our approach is consistent with the presently known phenomenology. We also argue that the sum rules derived from the bootstrap system can be used as a powerful tool to study hadron spectrum.

\section{REFERENCES}

1. S. Weinberg, Physica A 96, 327 (1979).

2. S. Weinberg, The Quantum Theory of Fields

(Cambrige University Press, Cambridge, 1999), vv. $1-2$.

3. A. Vereshagin, V. Vereshagin, and K. Semenov-Tian-Shansky, J. Math. Sci. 125, 144 (2005).

4. A. Vereshagin and V. Vereshagin, Phys. Rev. D 69, 025002 (2004).

5. K. Semenov-Tian-Shansky, A. Vereshagin, and V. Vereshagin, Phys. Rev. D 73, 025020 (2006).

6. K. Semenov-Tian-Shansky, A. Vereshagin and V. Vereshagin, Phys. Rev. D 77, 025028 (2008).

7. M. M. Nagels et al., Nucl. Phys. B109, 1 (1976); ibid B147, 189 (1979); O. Dumbrajs et al., Nucl. Phys. B216 277 (1983). 